\title{
Kounis syndrome, a disease to know: Case report and review of the literature
}

\author{
Edoardo Sciatti, Enrico Vizzardi, Dario S. Cani, Assunta Castiello, Ivano Bonadei, \\ Daria Savoldi, Marco Metra, Antonio D'Aloia \\ Cardiology Unit, University and Spedali Civili of Brescia, Italy
}

\begin{abstract}
The case deals with an anaphylactoid reaction to intravenous ampicillin/sulbactam resulting in cardiogenic syncope and myocardial damage. Symptoms and ECG modifications promptly disappeared after corticosteroids administration.

The Kounis syndrome is an acute coronary syndrome, including coronary spasm, acute myocardial infarction and stent thrombosis, resulting from an anaphylactic or anaphylactoid or allergic or hypersensitivity insult. First described in 1991, it can be caused by a lot of substances, particularly antibiotics. The management should be directed to both the allergic reaction and the myocardial damage.

The Kounis syndrome is a not rare disease that every physician should know because of the wideness of triggers and the possible fatal evolution if not promptly recognized.
\end{abstract}

\section{Case Report}

A 70-year old man came to the Emergency Department (ED) suffering of acute left-sided lumbar pain. He was affected by ischemic heart disease with mid-range left ventricular ejection fraction (LVEF). Few months before he had had an infero-lateral myocardial infarction complicated by apical endoventricular thrombosis and paroxysmal atrial fibrillation, treated with oral anticoagulant (Figure 1A). No documented history of anaphylactic/hypersensitivity reactions was known. He reported a history of recidivant hepatocarcinoma waiting for surgical resection of the III liver segment. He urgently underwent

Corresponding author: Edoardo Sciatti, Piazzale Spedali Civili, 125123 Brescia, Italy. Tel. +39.030.3995536 - Fax: +39.030.3995013. E-mail: edoardo.sc@tin.it

Key words: Kounis syndrome; anaphylactoid reaction; ampicillin/sulbactam; coronary artery; acute coronary syndrome.

Contributions: ES, DSCi, AC, wrote the paper; IB, MM, EV, ADA, DS, revised the paper.

Received for publication: 13 November 2017

Accepted for publication: 2 March 2018

(C) Copyright E. Sciatti et al., 2018

Tipografia PI-ME Editrice, Italy

Monaldi Archives for Chest Disease 2018; 88:898

doi: 10.4081/monaldi.2018.898

This article is distributed under the terms of the Creative Commons Attribution Noncommercial License (by-nc 4.0) which permits any noncommercial use, distribution, and reproduction in any medium, provided the original author(s) and source are credited. an abdominal CT scan revealing a bleeding retroperitoneal hematoma, treated with urgent left ileo-lumbar artery percutaneous embolization. Anticoagulation was stopped and he was hospitalized in the Surgery Department for monitoring and subsequent liver lesion surgical treatment. The hemodynamic parameters were stable (blood pressure 105/70 mmHg, hemoglobin $9.9 \mathrm{~g} / \mathrm{dL}$ ). As per protocol, he was administered ampicillin/sulbactam for infective prophylaxis. Suddenly during the infusion, the patient felt severe chest pain and presented a syncopal episode (blood pressure dropped to $60 / 40 \mathrm{mmHg}$, heart rate increased to $90 \mathrm{bpm}$ ). An electrocardiogram (ECG) was performed, showing sinus rhythm, inferior ST segment elevation, infero-lateral necrosis (Figure 1B). The patient was treated with infusion of corticosteroids (betametasone $4 \mathrm{mg}$ iv bolus), lisine acetylsalycilate (500 $\mathrm{mg}$ iv), saline solution and ephedrine (25 $\mathrm{mg}$ twice) and admitted to the Coronary Care Unit. Blood pressure and ECG rapidly normalized (F Figure 1C) and angor improved. A transthoracic echocardiogram revealed postero-lateral mid-distal hypokynesia, while inferior akynesia with fibrosis and basal posterior septal akynesia were confirmed (LVEF 47\%). Other causes of cardiogenic syncope were excluded, as well as endoventricular thrombosis. Troponin I values raised to 0.291 $\mathrm{ng} / \mathrm{mL}$. Ticagrelor and heparin were not administered considering the high hemorragic risk. The patient underwent coronary angiography, revealing diffuse moderate coronarosclerosis and a noncalcific plaque in the mid tract of the right coronary artery determining a $50 \%$ stenosis (Figure $2 \mathrm{~A}, \mathrm{~B}$ ). Medical therapy was continued, except for anticoagulant since the patient was re-transferred to the Surgery Department and underwent a successful liver resection without complications.

\section{Discussion}

The case described deals with a typical presentation of Kounis syndrome (KS), type II variant. The patient had an anaphylactoid reaction to betalactamics with syncope, hypotension and coronary vasospasm superimposed to coronarosclerosis. Symptoms and ECG modifications promptly disappeared after corticosteroids administration. No immunological tests to definitely prove the diagnosis were performed considering the patient's critical conditions after the cardiac event. However, the severe comorbidities of the patient, namely the liver cancer and the retroperitoneal hemorrhage, probably facilitated the onset of an immune-mediated reaction.

The KS is an acute coronary syndrome, including coronary spasm, acute myocardial infarction and stent thrombosis, resulting from an anaphylactic or anaphylactoid or allergic or hypersensitivity insult (clinical conditions characterized by mast cells activation with massive release of histamine). It is classified in three types according to the atheromatous state of the coronary arteries (Table 1).

Even in hypersensivity myocarditis myocardial damage is mediated by inflammation. The difference between the myocarditis and the KS is histological: in myocarditis there is presence of eosinophils, atypical 

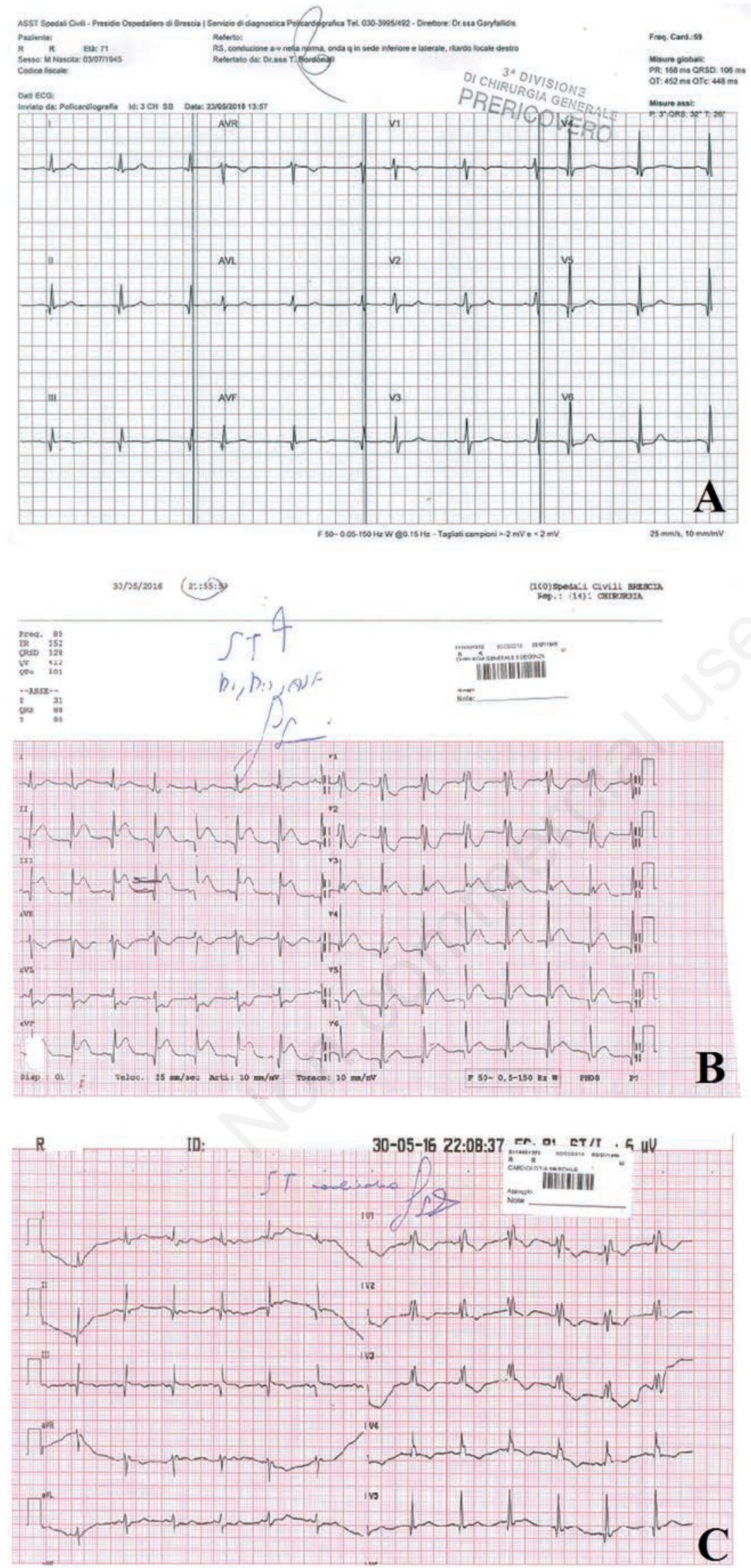

Figure 1. ECG evolution before (A), during (B) and after (C) Kounis syndrome. lymphocytes and giant cells in myocardial biopsy, whereas in KS these inflammatory cells are present in coronary intima, media and adventitia [1].

The first to talk about an allergic angina syndrome was Nicholas G. Kounis in 1991, but the association between an allergic reaction and an acute coronary syndrome had been described for the first time in 1950. The incidence is estimate at $7.9-9.6 / 100,000$ per year and the case-fatality rate seems to be $0.0001 \%$ [2].

KS has been observed in every age group, every race and geographical location. Although the syndrome is not rare, it is often not recognized and therefore not diagnosed nor well treated.

In this field, Lippi et al. analyzed TnI levels in all patients who were admitted to the ED with the diagnosis of anaphylaxis, angioedema or urticaria and they found them significantly increased in patients with anaphylactic or anaphylactoid syndrome compared with healthy controls [3]. This denotes that the heart and especially the coronary arteries constitute primary targets in anaphylaxis. Moreover, Cha et al. measuring both TnI levels and LVEF (assessed by echocardiopraphy) in 300 cases of anaphylaxis demonstrated that myocardial injury was present in $7.3 \%$ of patients [4].

The allergic activation process causes the release of inflammatory mediators such as histamine, platelet-activating factor, arachidonic acid products (leukotriene, thromboxane), neutral proteases and a variety of cytokines and chemokines. During an allergic or anaphylactic reaction, a lot of mediators can cause myocardial damage. In particular, the major role is played by the activation of mast cells secondary to the exposure to an antigen who is responsible for their degranulation, with release of inflammatory mediators and induced expression of lots of others factors (Table 2).

Various causes can induce KS: foods, drugs, environmental exposures. Since the syndrome was discovered, and in particular in recent years, several cases of KS have been described, secondary to allergic reaction to different substances. In Table 3 the most important cases in literature are reported. Clinically, KS manifests both with by signs and symptoms of an allergic reaction (headache, malaise, nausea, pruritus, vomiting, wheezing, skin rash, hypotension, diaphoresis, sweating) and those of an acute coronary syndrome (acute chest pain, dyspnoea, chest discomfort, palpitation, tachycardia 
or bradycardia, cardiorespiratory arrest). ECG signs are: ST segment elevation or depression, T-wave flattening and/or inversion, tachyarrhythmias or bradyarrhythmias. Laboratory tests often show leukocytosis with eosinofilia and increase in myocardial enzymes. Alterations in coronary angiography are different as different according to the KS type: spasm (type I), thrombosis (type II) or thrombosis on preexisting drug eluting stent (type III).

Management of KS is tricky because the treatment of the allergic reaction may worsen the myocardial damage (e.g., anti-H1 drugs can cause hypotension and coronary hypoperfusion epinephrine can increase myocardial oxygen consumption); vice versa the treatment of myocardial infarction cannot ameliorate the anaphylactic reaction. In this case, both conditions should be managed tailored on patient's clinical presentation. A recent review revealed intravenous steroids (76\%), nitroglycerin (47\%), H1-blockers (70\%), and H2-blockers (35\%) to be the most commonly used treatment options. A guide to approach the treatment options of this intricate syndrome is proposed in Figure 3.

To conclude, KS is a not rare disease that every physician should know because of the wideness of triggers and the possible fatal evolution if not promptly recognized. Our case report aims at underlying these two aspects, since the patient was receiving an antibiotic prophylaxis at the Surgery Department like thousands of people and necessitated of immediate resuscitation and coronary care unit stay in order to survive.
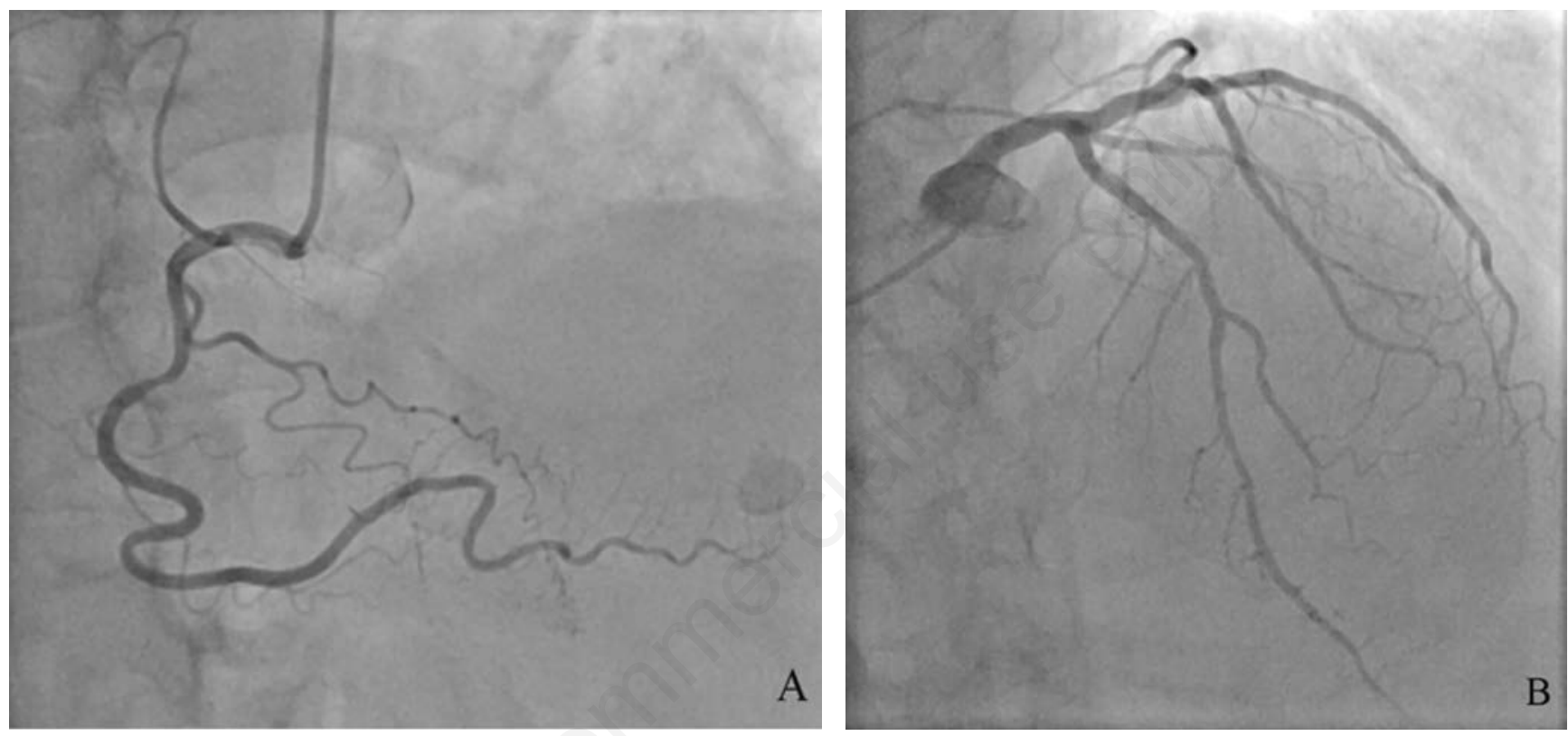

Figure 2. Angiography of right (A) and left (B) coronary arteries.

Table 1. Types of Kounis syndrome.

\begin{tabular}{ll} 
Types of Kounis syndrome & Description \\
Type I & Coronary artery spasms at the epicardial and/or microvascular levels in patients without coronary disease \\
\hline Type II & Plaque erosion or rupture in patients with pre-existing coronary atheromatous disease previously silent \\
\hline Type III & $\begin{array}{l}\text { Stent thrombosis in patients with previous implantation of coronary drug eluting stent, with demonstrated } \\
\text { presence of mast cell by Giemsa stain and eosynophils by ematoxylin-eosin stains }\end{array}$ \\
\hline
\end{tabular}

Table 2. Role of various inflammatory agents on coronary arteries.

\begin{tabular}{|c|c|}
\hline Inflammatory agent & Activity \\
\hline Histamine & $\begin{array}{l}\text { Coronary vasoconstriction by } \mathrm{Hl} \text { endothelial receptors, induction of tissue factor expression, and } \\
\text { platelets activation }\end{array}$ \\
\hline Tryptase & Activation of metalloproteinases with subsequent erosion of plaque collagen cap \\
\hline Chymase and Cathepsin D & Increased vasoconstriction by converting angiotensin I to angiotensin II \\
\hline Leukotrienes & Increased vasoconstriction \\
\hline Thromboxane & Platelets aggregation \\
\hline Platelets (Fc $\gamma$ RI, FC $\gamma$ RII, FCeRI and FCeRII) & Enhanced adhesion, activation and aggregation \\
\hline
\end{tabular}


Table 3. Case reports of Kounis syndrome in literature.

\begin{tabular}{|c|c|c|c|c|c|c|c|}
\hline Cause & Type & $\begin{array}{l}\text { Temporal } \\
\text { relation }\end{array}$ & LVEF & ECG & $\begin{array}{c}\text { Acute } \\
\text { treatment }\end{array}$ & $\begin{array}{l}\text { Regression of } \\
\text { CG alterations }\end{array}$ & $\begin{array}{l}\text { Prick test } \\
\text { or RAST + }\end{array}$ \\
\hline Anisakis simplex [5] & I & Yes & Moderate dysfunction & Anterior ST elevation & - & Yes & Yes \\
\hline Scombroid syndrome [6] & II & Yes & - & Infero-lateral ST changes & PTCA + stent & Yes & $\begin{array}{l}\text { Histamine } \\
\text { intoxication }\end{array}$ \\
\hline Losartan [7] & I & Yes & - & Lateral ST elevation & $\begin{array}{l}\text { Anti-allergic treatment, } \\
\text { inotropic support, IABP }\end{array}$ & Yes & Yes \\
\hline Gelofusine substance [8] & I & Yes & - & - & $\begin{array}{l}\text { Metaramirol, epinephrine, } \\
\text { steroids, antihistamine }\end{array}$ & - & Yes \\
\hline Ultrasound contrast [9] & II & Yes & - & Inferior ST changes & - & Yes & - \\
\hline Actinidia chinensis [10] & I & Yes & - & - & - & - & Yes \\
\hline Warble fly bite [11] & II & Yes & - & Anterio ST elevation & PTCA & - & - \\
\hline Corticosteroids [12] & I & Yes & - & - & - & Yes & - \\
\hline NSAIDS [13] & I & Yes & - & Bradycardia, biphasic T waves & Calcium channel blockers & - & - \\
\hline Autoimmune urticaria [14] & I & Yes & - & Anterio ST elevation & Cetirizine, methylprednisolone & Yes & Yes \\
\hline Metamizole [15] & I & Yes & Normal & ST elevation & Inotropic support, hydrocortisone & Yes & - \\
\hline Clarythromycin [16] & - & Yes & - & - & Sudden death & Sudden death & - \\
\hline Cefotaxime ev [17] & I & Yes & Normal & $\begin{array}{l}\text { ST depressionV3-V5, } \\
\text { ST elevation V1-aVR }\end{array}$ & $\begin{array}{l}\text { Standard protocol } \\
\text { for ACS, anti-H2 }\end{array}$ & - & - \\
\hline Ibuprofen [18] & I & Yes & - & Inferior ST elevation & $\begin{array}{l}\text { Trinitrine, intracoronary } \\
\text { epinephrine, IABP }\end{array}$ & - & - \\
\hline Unknown [19] & I & Yes & $20 \%$ & Infero-lateral ST elevation & Steroids, histamine blockers & Yes & - \\
\hline $\begin{array}{l}\text { Iopromide } \\
\text { (contrast agent) [20] }\end{array}$ & I & Yes & Normal & Inferior ST elevation & $\begin{array}{l}\text { Steroids, epinephrine, } \\
\text { vasodilators, anti-H2 }\end{array}$ & Yes & - \\
\hline Multiple bee stings [21] & II & Yes & $25 \%$ & Infero-lateral ST elevation & Steroids, epinephrine, PTCA & - & - \\
\hline Pea salad [22] & I & Yes & - & Inferior ST elevation & $\begin{array}{c}\text { Intracoronary nitroglycerine, } \\
\text { hydrocortisone }\end{array}$ & Yes & - \\
\hline Bee sting [22] & I & Yes & - & Inferior ST elevation & - & Yes & - \\
\hline $\begin{array}{l}\text { Amoxicillin/clavulanic } \\
\text { acid [23] }\end{array}$ & $\begin{array}{l}\text { Angiography } \\
\text { refused }\end{array}$ & Yes & Normal & ST elevation & $\begin{array}{c}\text { Epinephrine, hydrocortisone, } \\
\text { ACS therapy }\end{array}$ & - & - \\
\hline Bonsai [24] & - & Yes & Inferior hypokinesis & ST elevation & Thrombolytilc & Yes & - \\
\hline $\begin{array}{l}\text { Sulphur } \\
\text { hexafluoride [25] }\end{array}$ & III & Yes & - & Inferior ST elevation & $\begin{array}{c}\text { Hydrocortisone, } \\
\text { thrombus aspiration, PTCA }\end{array}$ & - & - \\
\hline Spider bite [26] & No angiography & Yes & $22 \%$ & ST segment depression & Treatment for HF & - & - \\
\hline During TAVR [27] & I & Yes & - & Inferior ST elevation & $\begin{array}{l}\text { Steroids, antihistamine, } \\
\text { epinephrine, nitrates }\end{array}$ & - & - \\
\hline Mushroom[28] & I & Yes & - & ST elevation DI aVL, VI-V4 & Intracoronary nitrates & Yes & Yes \\
\hline Anaesthesia [29] & I & Yes & - & Inferior ST elevation & - & - & - \\
\hline Capecitabine [30] & I & Yes & $40-50 \%$ & FV $\rightarrow$ Lateral ST elevation & - & Yes & - \\
\hline Midazolam [31] & I & Yes & 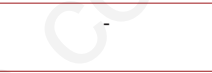 & Anterio ST depression & $\begin{array}{l}\text { Flumazenil, ACS therapy, } \\
\text { anti-H2, corticosteroids }\end{array}$ & - & - \\
\hline Wasp sting [32] & I & Yes & $40 \%$ & Tinversion & Epinephrine, hydrocorotisone & Yes & - \\
\hline Ciprofloxacine [33] & I & Yes & - & $\begin{array}{l}\text { ST elevation } \\
\text { on inferior }\end{array}$ & $\begin{array}{l}\text { Thrombolysis, } \\
\text { ACS therapy } \rightarrow \text { prednisone, } \\
\text { ranitidine, amlodipine }\end{array}$ & $\begin{array}{l}\text { Only after } \\
\text { second } \\
\text { treatment }\end{array}$ & - \\
\hline $\begin{array}{l}\text { After fish } \\
\text { consumption [34] }\end{array}$ & I & Yes & Normal & $\begin{array}{l}\text { ST elevation DI-aVL, } \\
\text { infero-lateral depression }\end{array}$ & Antihistamine, ACS therapy & - & - \\
\hline Bee sting [34] & I & Yes & - & $\begin{array}{c}\text { Inferior } \\
\text { ST elevation }\end{array}$ & $\begin{array}{c}\text { Epinephrine, hydrocorotisone, } \\
\text { dual antiplatelet therapy, heparin }\end{array}$ & Yes & - \\
\hline Wasp sting [35] & I & Yes & Normal & Biphasic T wave & Methilprednisolone, chlorpheniramine & ne & - \\
\hline Spider bite [36] & II & Yes & Normal & Inferior ST elevation & Thromboaspiration & - & - \\
\hline Ceftriaxone [37] & I & Yes & Normal & - & Intracoronary nytroglicerin & - & Negative \\
\hline Metimazole [38] & II & Yes & $\begin{array}{c}\text { Mild } \\
\text { dysfunction }\end{array}$ & $\begin{array}{l}\text { Inferior and anteriori } \\
\text { ST elevation }\end{array}$ & $\begin{array}{c}\text { Nitroglycerine } \\
\text { thromboaspiration, PTCA + BMS }\end{array}$ & - & - \\
\hline Amiodarone [39] & I & Yes & $\begin{array}{l}\text { Infero-lateral } \\
\text { hypokinesis }\end{array}$ & - & Epinephrine, dobutamine & - & - \\
\hline Tramadol [40] & I & Yes & - & $\begin{array}{l}\text { Cardiac arrest } \rightarrow \text { inferior } \\
\text { ST elevation }\end{array}$ & Epinephrine, nitroglycerine & - & - \\
\hline Celecoxib [41] & I & Yes & $\begin{array}{c}\text { Normal, } \\
\text { apical akynesis }\end{array}$ & Inferior ST elevation & $\begin{array}{l}\text { Diphenhydramine, albuterol, } \\
\text { methilprednisolone, heparin, } \\
\text { clopidogrel }\end{array}$ & Yes & - \\
\hline $\begin{array}{l}\text { After rupture of } \\
\text { echinoccocal cyst [42] }\end{array}$ & I & Yes & - & ST elevation & Chlorphenamine, hydrocortisone & Yes & - \\
\hline Tramadol ev [43] & I & Yes & $55 \%$ & $\begin{array}{l}\text { ST elevation DI-aVL, } \\
\text { depression DIII-V3-V5 }\end{array}$ & ACS therapy & Yes & - \\
\hline Clopidogrel [44] & I & Yes & - & Inferior ST elevation & Dexamethasone & - & - \\
\hline
\end{tabular}




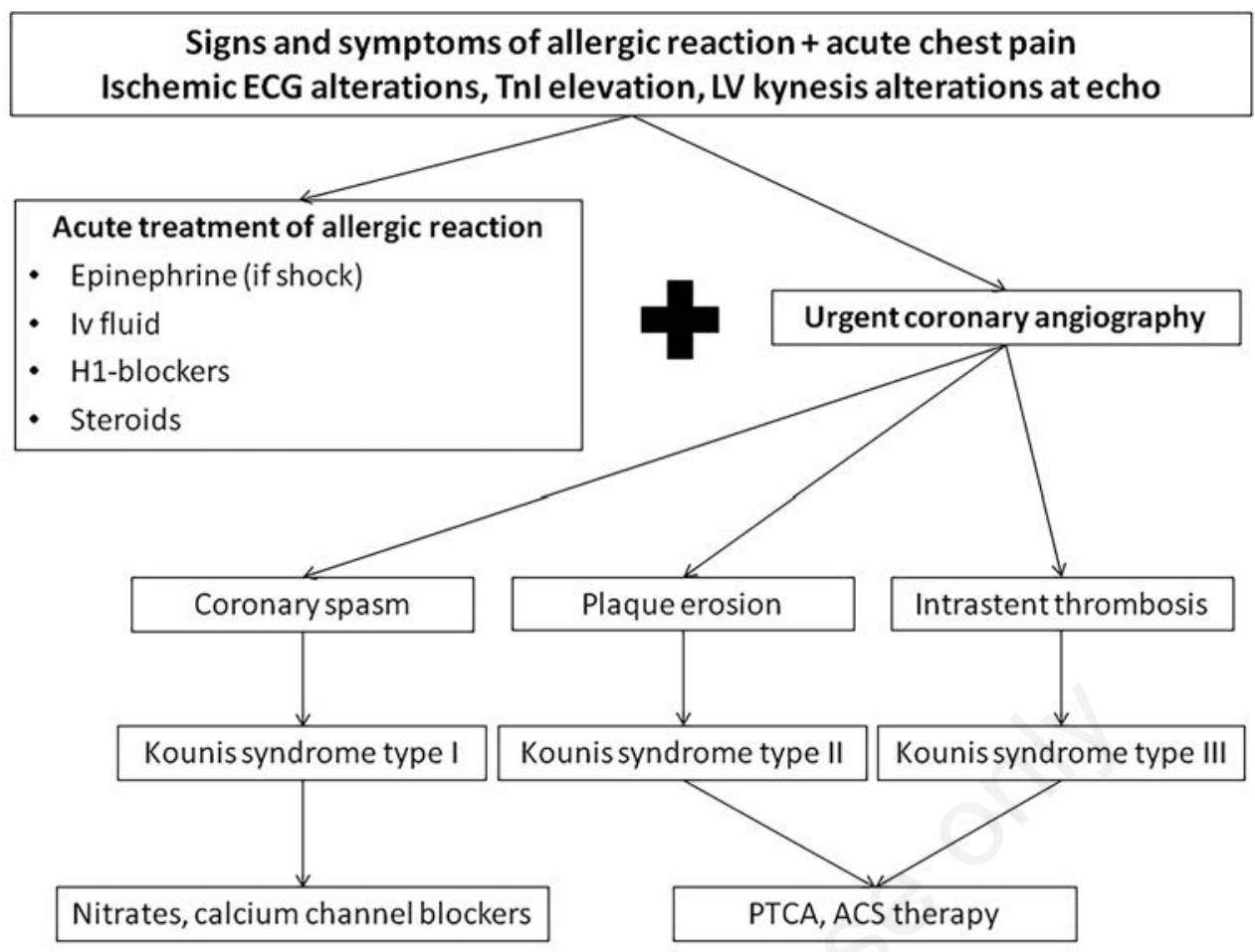

Figure 3. Proposed urgent treatment options of Kounis syndrome.

\section{References}

1. Kounis NG, Mazarakis A, Tsigkas G, et al. Kounis syndrome: a new twist on an old disease. Future Cardiol 2011;7:805-24.

2. Helbling A, Hurni T, Mueller UR, Pichler WJ. Incidence of anaphylaxis with circulatory symptoms: A study over a 3-year period comprising 940000 inhabitants of the Swiss Canton Bern. Clin Exp Allergy 2004;34:285-90.

3. Lippi G, Buonocore R, Schirosa F, Cervellin G. Cardiac troponin I is increased in patients admitted to the emergency department with severe allergic reactions. A case-control study. Int J Cardiol 2015;194:68-9.

4. Cha YS, Kim H, Bang MH, et al. Evaluation of myocardial injury through serum troponin I and echocardiography in anaphylaxis. Am J Emerg Med 2016;34:140-4.

5. Barbarroja-Escudero J, Rodriguez-Rodriguez M, Sanchez-Gonzalez MJ, et al. Anisakis simplex: a new etiological agent of Kounis syndrome. Int J Cardiol 2013;1676:e187-9.

6. Coppola G, Caccamo G, Bacarella D, et al. Vasospastic angina and scombroid syndrome: a case report. Acta Clin Belg 2012;67:222-5.

7. Josefsson J, Fröbert 0. Losartan-induced coronary artery spasm. BMJ Case Rep 2012;2012. pii: bcr2012006252.

8. Shah G, Scadding G, Nguyen-Lu N, et al. Peri-operative cardiac arrest with ST elevation secondary to gelofusin anaphylaxis - Kounis syndrome in the anaesthetic room. Int J Cardiol 2013;164:e22-6.

9. Van Ginkel AG, Sorgdrager BJ, de Graaf MA, et al. ST-segment elevation associated with allergic reaction to echocardiographic contrast agent administration. Neth Heart J 2013;15:725-6.

10. Guler Y, Kalkan S, Esen AM. An extremely rare trigger of Kounis syndrome: Actinidia chinensis. Int J Cardiol 2014;172:24-5.
11. Karadeniz M, Akyel A, Celik IE, et al. An unusual etiology of Kounis syndrome; warble fly. Indian Heart J 2013;65:358-359.

12. Arslan Z, Iyisoy A, Tavlasoglu M. Acute myocardial infarction after prednisolone administration for the treatment of anaphylaxis caused by a wasp sting. Cardiovasc J Africa 2013;24:e4-e6.

13. Rayner-Hartley E, Chou A, Saw J, Sedlak T. A case of Kounis type I in a young woman with Samter's triad. Can J Cardiol 2016;32:1261.e1-1261.e3.

14. Erxun K, Wei L, Shuying Q. Kounis syndrome caused by chronic autoimmune urticaria: A case report. J Emerg Med 2016;50:37-40.

15. Juste JF, Garces TR, Enguita RG, et al. Cardiac complications in a metamizole-induced type I Kounis syndrome. Braz J Anesthesiol 2016;66:194-6.

16. Kounis NG, Kounis GN, Soufras GD, et al. Postmortem diagnosis of drug-induced anaphylactic death: Kounis syndrome and hypersensitivity myocarditis are the likely culprit in death of severe anaphylactic reactions. J Forensic Leg Med 2016;40:40-1.

17. Venkateswararao S, Rajendiran G, Sundaram RS, Mounika G. Kounis syndrome secondary to intravenous cephalosporin administration. J Pharmacol Pharmacother 2015;6:225-7.

18. Ihdayhid AR, Rankin J. Kounis syndrome with Samter-Beer triad treated with intracoronary adrenaline. Catheter Cardiovasc Interv 2015;86:E263-7.

19. Arora S, Patel R, Fadila M, Wool K. The atopic heart: a curious case of coronary hypersensitivity. Neth J Med 2016;74:130-2.

20. Oh KY, In YN, Kwack CH, et al. Successful treatment of Kounis syndrome type I presenting as cardiac arrest with ST elevation. Chin Med J (Engl) 2016;129:626-7.

21. Pelli JR Jr, Wieters JS, Firozgary B, Montalvo T. Multiple bee stings resulting in ST elevation myocardial infarction (the Kounis syndrome). Proc (Bayl Univ Med Cent) 2016;29:298-300. 
22. Memon S, Chhabra L, Masrur S, Parker MW. Allergic acute coronary syndrome (Kounis syndrome). Proc (Bayl Univ Med Cent) 2015;28:358-62.

23. Ralapanawa DM, Kularatne SA. Kounis syndrome secondary to amoxicillin/clavulanic acid administration: a case report and review of literature. BMC Res Notes 2015;8:97.

24. İnci S, Aksan G, Doğan A. Bonsai-induced Kounis Syndrome in a young male patient. Anatol J Cardiol 2015;15:952-954.

25. Portero-Portaz JJ, Córdoba-Soriano JG, Gallego-Page JC. Type III Kounis syndrome after administration of an echocardiography contrast agent. Eur Heart J Acute Cardiovasc Care 2016. pii: 2048872616655943. [Epub ahead of print].

26. Yaman M, Mete T, Ozer I, Yaman E, Beton 0. Reversible myocarditis and pericarditis after black widow spider bite or Kounis syndrome? Case Rep Cardiol 2015;2015:768089.

27. Benedetto D, Agostoni P, de Waal E, Stella PR. Kounis syndrome with cardiogenic shock during transfemoral transcatheter aortic valve replacement. Coron Artery Dis 2015;26:726-7.

28. Tepetam FM, Dağdeviren B, Bulut İ, et al. A patient with mushroom allergy; a new etiological agent of Kounis syndrome. Tuberk Toraks 2016;64:171-4.

29. Goto K, Kasama S, Sato M, Kurabayashi M. Myocardial scintigraphic evidence of Kounis syndrome: what is the aetiology of acute coronary syndrome? Eur Heart J 2016;37:1157.

30. Kido K, Adams VR, Morehead RS, Flannery AH. Capecitabine-induced ventricular fibrillation arrest: Possible Kounis syndrome. J Oncol Pharm Pract 2016;22:335-40.

31. Ateş $\mathrm{AH}, \mathrm{Kul} \mathrm{S}$. Acute coronary syndrome due to midazolam use: Kounis syndrome during a transurethral prostatectomy. Turk Kardiyol Dern Ars 2015;43:558-61.

32. Anandan PK, Hanumanthappa NB, Bhatt P, Cholenahally MN. Allergic angina following wasp sting: Kounis syndrome. Oxf Med Case Reports 2015;2015:306-8.
33. Ntuli PM, Makambwa E. Kounis syndrome. S Afr Med J 2015; $105: 878$

34. Katsanou K, Karagiannidis I, Oikonomou G, Kounis NG. Kounis syndrome: Report of 3 cases. Int J Cardiol 2015;197:222-3.

35. Venturini E, Marabotti C, Magni L, et al. Myocardial bridge as a trigger of Kounis syndrome. Int J Cardiol 2016;202:87-9.

36. Cervellin G, Neri G, Lippi G, et al. Kounis syndrome triggered by a spider bite. A case report. Int J Cardiol 2016;207:23-4.

37. Barbarroja-Escudero J, Sánchez-González MJ, Antolín-Amérigo D, et al. Kounis syndrome induced by cefditoren pivoxil. Int J Cardiol 2016;207:112-4.

38. Lorca R, Velasco E, Madera J, et al. Kounis syndrome: Identifying the trigger. Int J Cardiol 2016;209:179-80.

39. Cheung M, Seres T, Cleveland J, Kounis NG. Kounis syndrome, a coronary hypersensitivity disorder: A rare case of amiodarone-induced coronary vasospasm and simultaneous peripheral vasodilation intraoperatively. Int J Cardiol 2016;218:267-8.

40. Kim HI, Cha KC, Cha YS, et al. A subset of type I variant Kounis syndrome: Allergic angina syndrome and persistent presence of coronary spasm. Int J Cardiol 2016;223:959-61.

41. Regis AC, Germann CA, Crowell JG. Myocardial infarction in the setting of anaphylaxis to celecoxib: A case of Kounis syndrome. J Emerg Med 2015;49:e39-43.

42. Mirijello A, Pepe G, Zampiello P, et al. A male patient with syncope, anaphylaxis, and ST-elevation: Hepatic and cardiac echinococcosis presenting with Kounis syndrome. J Emerg Med 2016;51:e73-7.

43. Gormel S, Ege T, Koklu M, Celik M, Yuksel UC. Acute lateral myocardial infarction secondary to tramadol-induced Kounis syndrome. J Cardiothorac Vasc Anesth 2015;29:1599-602.

44. Liping Z, Bin H, Qiming F. An extraordinary case associated with an allergic reaction to clopidogrel: Coronary artery spasm or Kounis syndrome. Heart Lung Circ 2015;24:e180-3. 